\title{
Lithium-ion batteries Remaining Useful Life Prediction Method Considering Recovery Phenomenon
}

\author{
Zhenyu Zhang ${ }^{1,2,3}$, Dongxu Shen ${ }^{1,2,3}$, Zhen Peng ${ }^{4}$, Yong Guan ${ }^{1,2,3}$, Huimei Yuan ${ }^{1,2,3}$, Lifeng Wu ${ }^{1,2,3, *}$ \\ ${ }^{1}$ College of Information Engineering, Capital Normal University, Beijing 100048, China; \\ ${ }^{2}$ Beijing Key Laboratory of Electronic System Reliability Technology, Capital Normal University, \\ Beijing 100048, China \\ ${ }^{3}$ Beijing Advanced Innovation Center for Imaging Technology, Capital Normal University, Beijing \\ 100048, China \\ ${ }^{4}$ Information Management Department, Beijing Institute of Petrochemical Technology, Beijing 10217 , \\ China \\ *E-mail: wulifeng@cnu.edu.cn
}

doi: $10.20964 / 2019.08 .61$

Received: 4 April 2019 / Accepted: 30 May 2019 / Published: 30 June 2019

\begin{abstract}
Estimation of lithium battery remaining useful life (RUL) is the key to lithium battery health management. In the process of intermittent discharging lithium batteries, the recovery phenomenon will have a relatively large impact on the life of lithium batteries. However, in the nowadays research on the RUL of lithium batteries, the recovery phenomenon in the process of intermittent discharging lithium batteries is rarely taken into account. In this paper, a degradation model and RUL prediction method of lithium batteries considering the recovery effect are proposed. Firstly, under the framework of Wiener process theory, the RUL degradation model without recovery effect is established. Then, by considering the effect of lithium battery recovery on remaining useful life, the RUL degradation model with recovery effect is derived. And in the sense of the first passage time, theoretically, the RUL distribution of lithium batteries with recovery effects or no recovery effects is derived. Furthermore, the unknown parameters of the model are estimated by the method of maximum likelihood estimation. Finally, the designed experiment obtained the degradation data of 18650 lithium battery, and the method in this paper was applied to verify the effectiveness of the proposed method.
\end{abstract}

Keywords: remaining useful life ; recovery phenomenon; lithium batteries; degradation modeling

\section{FULL TEXT}

(C) 2019 The Authors. Published by ESG (www.electrochemsci.org). This article is an open access article distributed under the terms and conditions of the Creative Commons Attribution license (http://creativecommons.org/licenses/by/4.0/). 